\title{
Reactive Oxygen Species-Mediated Inflammation and Apoptosis in Hand-Foot Syndrome Induced by PEGylated Liposomal Doxorubicin
}

This article was published in the following Dove Press journal: International Journal of Nanomedicine

\author{
Xiaolin $\mathrm{Hu}^{\mathrm{l}, 2}$ \\ Mengmeng Dong ${ }^{2}$ \\ Xiao Liang $^{2}$ \\ Ziling Liu' \\ Quanshun Li iD ${ }^{2}$ \\ 'Cancer Center, The First Hospital of Jilin \\ University, Changchun 130012, People's \\ Republic of China; ${ }^{2}$ Key Laboratory for \\ Molecular Enzymology and Engineering of \\ Ministry of Education, School of Life \\ Sciences, Jilin University, Changchun \\ |300| 2, People's Republic of China
}

Correspondence: Quanshun Li; Ziling Liu Tel +86-43I-85I 5520 I

Fax +86-43I-85I55200

Email quanshun@jlu.edu.cn; ziling@jlu.

edu.cn
Background: Doxil ${ }^{\circledR}$ (PEGylated liposomal doxorubicin, PLD) has been widely used in cancer treatment due to its excellent therapeutic efficacy, but it can simultaneously cause severe adverse effects such as hand-foot syndrome (HFS). To date, the pathophysiologic mechanism of HFS development induced by PLD administration has not been well understood.

Materials and Methods: The histological features of skin lesion in PLD-induced HFS model were characterized by hematoxylin and eosin (H\&E) staining and picrosirius red staining, and the induction of inflammation and apoptosis in the epidermal layer was detected by immunohistochemical and TUNEL staining. Moreover, the generation of reactive oxygen species (ROS) was determined to elucidate the potential mechanism of skin lesion in the development of HFS.

Results: The administration of PLD has been demonstrated to induce the histological damage of skin tissues including the destruction of collagen fibers and the induction of severe inflammation and apoptosis of epidermal cells. The mechanism was probably attributed to the accumulation of PLD in the skin tissues during the long-term circulation and further the induction of ROS to cause the oxidative damage of keratinocytes owing to the sustained release of doxorubicin from PLD.

Conclusion: The ROS generation induced by the administration of PLD has been identified to be a crucial factor in the development of HFS, which could be used as a potential therapeutic target to alleviate the HFS symptom of PLD administration.

Keywords: PEGylated liposomal doxorubicin, hand-foot syndrome, reactive oxygen species, skin lesion

\section{Introduction}

Doxil $^{\circledR}$ is a formulation of PEGylated liposomal doxorubicin (PLD), in which doxorubicin (DOX) is encapsulated in sterically stabilized liposomes containing lipid derivatives of hydrophilic polyethylene glycol. ${ }^{1-4}$ In comparison to DOX, PLD could reduce the drug elimination by reticuloendothelial system (RES) due to the PEGylation of liposomes, further leading to the change of pharmacokinetic profile such as the reduced biodistribution volume, extended circulation time and enhanced drug penetration into tumors. ${ }^{5}$ Owing to these pharmacokinetic advantages, PLD could significantly inhibit tumor growth and improve the survival ratio of patients in the clinic. ${ }^{6}$ More importantly, the use of PLD showed approximately 3-fold lower risk of developing congestive heart failure compared to patients receiving the same dosage 
of DOX. ${ }^{7,8}$ Nevertheless, PLD has been found to possess other adverse effects such as stomatitis and hand-foot syndrome (HFS). ${ }^{9-11}$

HFS, also called palmar-plantar erythrodysesthesia (PPE), is a common dermatological side effect induced by some chemotherapeutic agents such as capecitabine, 5-fluorouracil and PLD. ${ }^{12-15}$ Particularly, the incidence rate of HFS could be up to $80 \%$ when the patients received a high dosage of PLD. ${ }^{13}$ The clinical features of HFS typically include paresthesia and stinging in the first 12 days after the application of chemotherapeutics and further evolve into severe skin lesions such as blister, peeling, crusting, erosion, ulceration and epidermal necrosis. ${ }^{14}$ The damaged skin tissues are histologically characterized by the hyperkeratosis of stratum corneum, the spongiosis of spinous layer, the focal vacuole-like degeneration of basal layer, the perivascular lymphocytic infiltration and the melanin deposition. ${ }^{15}$ Following the intravenous administration, PLD can accumulate in the deep and superficial exocrine glands and further penetrate into the stratum corneum of extremities due to the extravasation in the long-term circulation. ${ }^{16}$ Additionally, the PEGylation feature of PLD promotes the excretion of drugs via sweat. ${ }^{17}$ Subsequently, DOX molecules released from PLD will accumulate in skin tissues and lead to the oxidative stress damage of biomolecules in the corneum cells, thereby causing severe skin lesion. ${ }^{18,19}$ However, the detailed mechanism of skin damage in the PLD-induced HFS still remains unclear. Thus, it is urgent and necessary to understand the pathological mechanism of HFS induced by the administration of PLD, which is beneficial to improve the efficacy and safety of nanomedicine.

In the present study, the histological features of skin injury in the PLD-induced HFS model were systematically characterized, and meanwhile the level of proinflammatory cytokines and the apoptotic effect were detected in the epidermal layer. Moreover, the generation of reactive oxygen species (ROS) induced by the administration of PLD was measured to elucidate the potential mechanism of skin lesion in the development of HFS.

\section{Materials and Methods}

\section{Materials}

PLD was purchased from CSPC Pharmaceutical Group Ltd. (Hebei, China). DOX (hydrochloride form) was provided by Huafeng Chemical Co., Ltd. (Beijing, China). Hematoxylin, eosin and picrosirius red were purchased from Solarbio (Beijing, China). 3-(4,5-Dimethylthiazol2-yl)-2,5-diphenyltetrazolium bromide (MTT) was obtained in Amersco (Solon, OH). Fetal bovine serum (FBS) was provided by Kangyuan Co. (Beijing, China), and Dulbecco's modified Eagle's medium (DMEM) was purchased from Gibco (Grand Island, NE, USA). The primary antibodies against mouse IL- 6 and IL- $1 \beta$ were purchased from Abcam (Shanghai, China). The Annexin V-FITC/PI apoptosis detection kit was purchased from Vazyme Co. (Nanjing, China). The ROS detection kit and TUNEL apoptosis assay kit were provided by Beyotime (Jiangsu, China). All other reagents were of the highest grade commercially available and used as received.

\section{Cell Culture}

Human immortalized keratinocyte cell line HaCaT was obtained from the Shanghai Institute of Cell Bank (Shanghai, China) and cultured in DMEM containing $10 \% \mathrm{FBS}, 100 \mathrm{U}$ penicillin and $100 \mathrm{U}$ streptomycin at an atmosphere of $5 \% \mathrm{CO}_{2}$.

\section{In vitro Cytotoxicity Analysis}

The cytotoxicity of DOX and PLD was assessed by MTT method according to our previous reports. ${ }^{20,21}$ Briefly, HaCaT cells were seeded in 96-well plates at a density of 8000 cells/well and cultured as described above. After $12 \mathrm{~h}$, the cells were treated with various concentrations of DOX and PLD for additional 24 or 48 h. Then $20 \mu \mathrm{L}$ of MTT solution $(5 \mathrm{mg} / \mathrm{mL}$ in PBS) was added into each well, and the cells were incubated for 4 h. Subsequently, dimethyl sulfoxide $(200 \mu \mathrm{L})$ was used to dissolve the formed formazan crystal, and the absorbance at $492 \mathrm{~nm}$ was measured by a GF-M3000 microplate reader (Shandong, China) to calculate the cell viability.

\section{Cell Apoptosis Analysis}

The $\mathrm{HaCaT}$ cells were cultured in 6-well plates at an initial density of $2.5 \times 10^{5}$ cells/well, and then incubated with DOX or PLD at a drug concentration of $2.0 \mu \mathrm{M}$. After the incubation for $48 \mathrm{~h}$, the cells were subjected to staining at $4^{\circ} \mathrm{C}$ for $30 \mathrm{~min}$ according to the instructions of Annexin V-FITC/PI apoptosis detection kit. Finally, the cell apoptosis was analyzed by CytoFLEX flow cytometer (Beckman Coulter Inc., Brea, CA, USA). 


\section{In vitro TUNEL Staining Assay}

The cell culture and the treatment with DOX or PLD were carried out as described in the section "Cell apoptosis analysis", then the cells were rinsed with PBS three times and treated with $0.1 \%$ Triton $\mathrm{X}-100$ solution at room temperature for $2 \mathrm{~min}$. After being rinsed with PBS, the cells were treated with TUNEL reagent according to the manufacturer's protocol. Finally, the cells were rinsed with PBS again and observed on an Olympus IX73P1F fluorescence microscopy (Tokyo, Japan).

\section{ROS Level Analysis in $\mathrm{HaCaT}$ Cells}

The ROS level in HaCaT cells was measured according to the protocol in the ROS assay kit. Briefly, the cell culture and the treatment with DOX or PLD were performed as described in the section "Cell apoptosis analysis". Then the harvested cells were washed with PBS and incubated with DCHF-DA fluorescence probe at $37^{\circ} \mathrm{C}$ for $30 \mathrm{~min}$ in the dark. Finally, the cells were rinsed with PBS and detected by CytoFLEX flow cytometer (Beckman Coulter Inc.).

\section{Establishment and Characterization of HFS Animal Models}

All the animal studies were conducted according to the "Guide for the Care and Use of Laboratory Animals" (8th edition, International Publication No: 978-0-309-15,400-0) and approved by the Institutional Animal Ethics Committee of Jilin University (license No. SCXK-(JI) 2014-0012). Sixweek-old Wistar female rats were purchased from Vital River Laboratory Animal Technology Co., Ltd. (Beijing, China). Before use in this study, all rats were provided with standard diet and water for one week. To construct the HFS animal models, DOX or PLD $(10 \mathrm{mg} / \mathrm{kg})$ was intravenously injected into the Wistar rats once every 3 days. The onset of HFS was recognized as the redness of the skin. The rats were weighed every day and sacrificed on day 10 . The paws of rats were photographed after 1 minute and 10 days since the administration. Subsequently, the skin of the damaged paws and main organs were collected, fixed in $10 \%$ formalin solution, dehydrated and then embedded in paraffin. The embedded tissues were sectioned into $4-\mu \mathrm{m}$ slides, which were stained with hematoxylin and eosin (H\&E) solution and observed by Olympus IX73P1F fluorescence microscopy. Meanwhile, to detect the dermal collagen fibers in the skin, the sections were stained with picrosirius red and then photographed with a NIKON Eclipse ci polarized light microscopy (Tokyo, Japan).

\section{TUNEL Staining of Skin Tissues}

The sectioned skin tissues were first incubated with $0.25 \%$ of proteinase $\mathrm{K}$ solution at room temperature for $15 \mathrm{~min}$, and washed with PBS twice. Then $0.1 \%$ Triton X-100 solution was added to completely cover the sections which were incubated at room temperature for $2 \mathrm{~min}$. Afterwards, the sections were treated with TUNEL solution and covered with coverslips according to the manufacturer's instructions. Finally, the sections were placed in the dark for $60 \mathrm{~min}$ and observed on an Olympus IX73P1F fluorescence microscopy (Tokyo, Japan).

\section{Immunohistochemical (IHC) Staining of Pro-Inflammatory Cytokines}

The slides of skin tissues with the thickness of $4 \mu \mathrm{m}$ were deparaffinized with xylene and rehydrated with ethanol. The slides were then treated with citric acid buffer $(\mathrm{pH}$ 6.0) and heated in a microwave oven for $20 \mathrm{~min}$ to achieve the antigen retrieval. After cooling to room temperature, the slides were incubated with primary antibodies (dilution of $1: 200$ ) at $4^{\circ} \mathrm{C}$ overnight and detected using a horseradish peroxidase (HRP) conjugated compact polymer system. Diaminobenzidine (DAB) was used as the chromogen to observe the immune reactive cells, and the sections were then stained with hematoxylin. The images of tissue sections were captured on an Olympus IX73P1F fluorescence microscopy.

\section{ROS Level Analysis in Skin Tissues}

To measure the ROS level in the skin tissues, the skin isolated from the hind paws of rats was cut into tiny pieces and subsequently placed on the nylon net. The skin tissues were simultaneously rubbed and rinsed with PBS to collect the suspended cells. The obtained cells were treated according to the ROS assay kit's instructions, and the ROS level was detected by CytoFLEX flow cytometer (Beckman Coulter Inc.).

\section{Statistical Analysis}

Data were expressed as mean value \pm SD using GraphPad Prism 6 software (San Diego, CA, USA). One-way ANOVA analysis was used to compare the statistical differences between experimental groups and control group (n.s., not significant; ${ }^{*} p<0.05 ;{ }^{*} p<0.01$ ). 


\section{Results and Discussion}

Skin Lesions in the PLD-Induced HFS

\section{Model}

The PLD-induced HFS model was established by the intravenous administration of $10 \mathrm{mg} / \mathrm{kg}$ PLD in the female Wistar rats. As shown in Figure 1, compared with the rats receiving DOX, obvious erythema could be observed in both forepaws and hindpaws of the rats after the administration of PLD for $1 \mathrm{~min}$. Though this phenomenon preserved for the following $30 \mathrm{~min}$ and gradually ameliorated overnight, more severe skin injury could be found in the rats receiving PLD after 10 days, whose limbs turned to erythema, swelling and ulceration symptoms. Additionally, the paws appeared to have redness and dryness when the rats were treated with PLD, much similar to the HFS symptoms in humans. However, no skin lesions were observed in the rats receiving DOX. These results demonstrated that the HFS animal models have been successfully established via the intravenous injection of PLD, in which PLD could cause severe skin damage of rats' limbs. After the intravenous injection of PLD, the PEGylation could significantly increase the half-life of drugs and meanwhile avoid the rapid elimination by RES system, thereby changing the intrinsic biodistribution of free drugs or nonPEGylated nanomedicine. ${ }^{16}$ Owing to the extended circulation time, obvious accumulation of PLD in the limbs could be observed which would cause the extravasation of PLD from the capillary and the further distribution in the deep and superficial exocrine glands. In addition, the PEGylation not only promoted the sweat secretion but also accelerated the drug release in the skin, which could gradually penetrate into the stratum corneum of the hands and feet, causing damage to the skin. ${ }^{22,23}$

Subsequently, the histological analysis of paws' skin tissues isolated from the HFS rats was performed by H\&E staining and picrosirius red staining. As shown in Figure 2, compared with normal rats, the epidermal layer of rats' paws become thinner after treatment with PLD, owing to the induction of ulceration. Moreover, the PLD treatment led to the disorderly arranged epithelial cells, the reduction or even diminishment of stratum granulosum, and the decreased number of cells in basal cell layer and stratum spinosum. Further, picrosirius red staining was conducted and morphometric images were analyzed to determine whether the PLD administration could affect the collagen network in skin tissues. As shown in Figure S1, more area with red color could be observed in the epidermal layer of normal rats' skin tissues, while limited red color was obtained in the PLD treatment group, indicating that the administration of PLD decreased the collagen content in the paws' skin. Besides, the collagen type could be identified according to the specific colors under polarized light, in which yellow-red color represented type I collagen, whereas type III collagen exhibited green color (Figure 2).

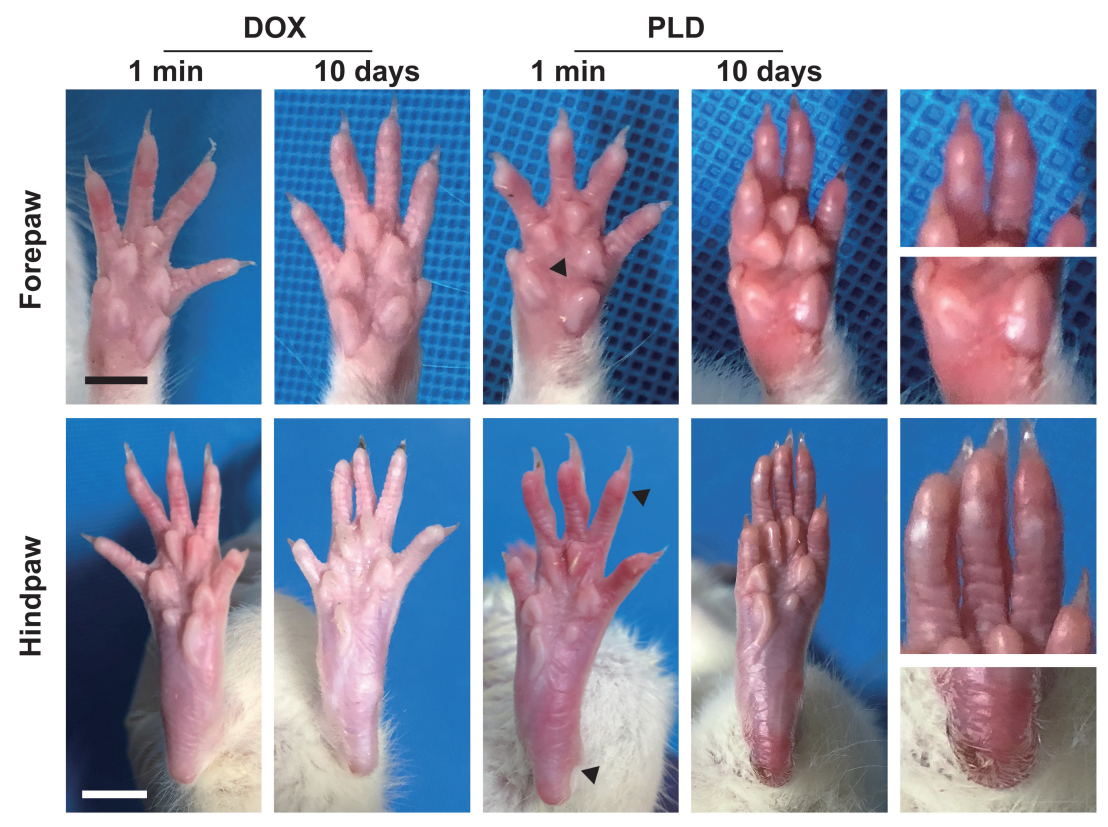

Figure I The paws of rats after the intravenous injection of DOX and PLD at a dose of $10 \mathrm{mg} / \mathrm{kg}$. Black arrows indicated the swelling and redness of the damaged skins. Scale bar: $1 \mathrm{~cm}$. 


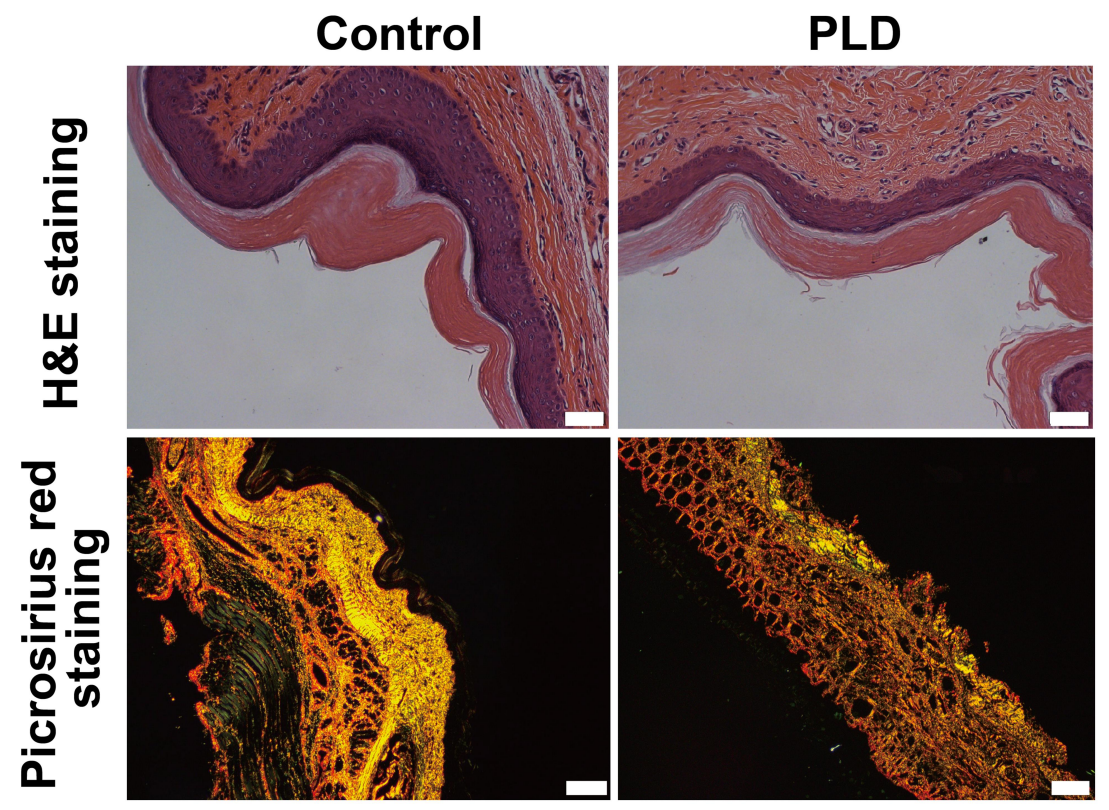

Figure 2 H\&E staining and picrosirius red staining (under polarized light microscopy) of the paws' skin tissues. Scale bar: $50 \mu \mathrm{m}$.

Apparently, a large proportion of type I and III collagen could be observed in normal rat's skin tissues. However, the collagen networks appeared to be diminished in the rats treated with PLD, with thinner and shorter fibers than those in normal rats. This phenomenon revealed that the PLD treatment could break and disarrange the collagen fibers to disrupt the skin architecture. In addition, the toxicity profile of PLD was evaluated by monitoring the changes of body weight and the histological analysis of main organs. As shown in Figure S2, the body weight of rats gradually decreased following the administration of PLD, indicating that PLD could cause obvious toxicity to rats. However, there were no significant pathological changes in the main organs after the intravenous injection of PLD for three times (Figure S3). Particularly, PLD did not cause obvious damage to the heart tissue, while DOX was widely accepted to be cardiotoxic. Thus, these results demonstrated that the unique pharmacokinetic profile of PLD led to a higher accumulation of PLD in the skin tissues and a relatively lower distribution in the main organs, facilitating the emergence of HFS and the decreased toxicity on the other organs.

\section{Induction of Inflammation and Apoptosis in the Skin Tissues by PLD}

The expression level of pro-inflammatory cytokines in the rats' palmar-toe skin was performed by immunohistochemistry (IHC). As shown in Figure 3, there was no expression of pro-inflammatory cytokines IL-6 and IL-
$1 \beta$ in normal rats. In contrast, the skin section obtained from the rats receiving PLD exhibited obvious expression of IL- 6 and IL-1 $\beta$ in the epidermal layers, indicating that the PLD administration could induce significant inflammatory effect in the metacarpophalangeal skin. The pro-inflammatory cytokines secreted in the skin tissues could potentially stimulate the lymphocytes residing around postcapillary venules in the skin to produce more cytokines, further enhancing the inflammatory damage. Moreover, the sustained inflammation could dramatically deteriorate the function of collagen fibers. ${ }^{24,25}$

Besides, the cell apoptotic effect of skin was evaluated by TUNEL staining assay (Figure 4). Clearly, compared with normal rats, obvious green fluorescence could be obtained in the skin sections isolated from the rats receiving PLD, indicating that the PLD treatment enhanced the cell apoptosis in the epidermal layer. Subsequently, to further understand the phenomenon of cell apoptosis, the cell viability was evaluated by MTT method using the human keratinocyte cell line $\mathrm{HaCaT}$ as a model. As shown in Figure S4, the cell proliferation of $\mathrm{HaCaT}$ has been significantly inhibited by the treatment with DOX and PLD for 24 and $48 \mathrm{~h}$ in a concentrationdependent manner. Additionally, the inhibition of cell proliferation was more apparent at $48 \mathrm{~h}$, where PLD could decrease the cell viability to $76.3 \%$ at a concentration of $4.0 \mu \mathrm{M}$. Notably, compared with 


\section{IL-6}
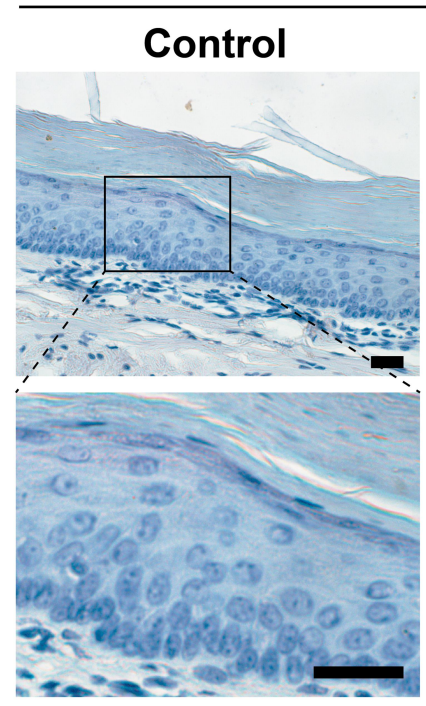
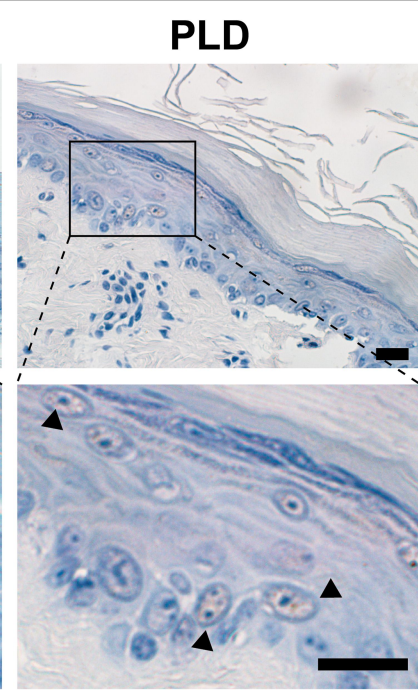

IL-1 $\beta$

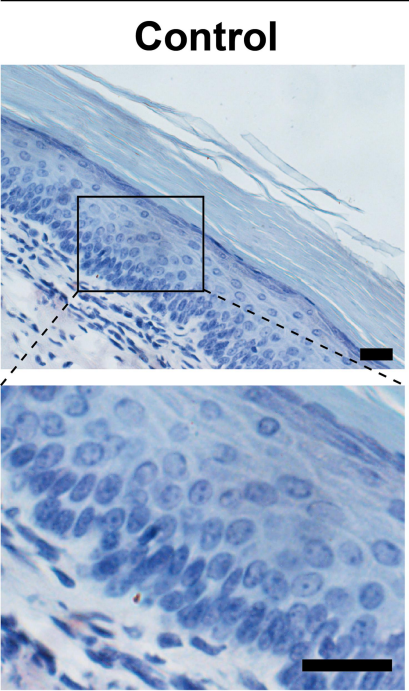

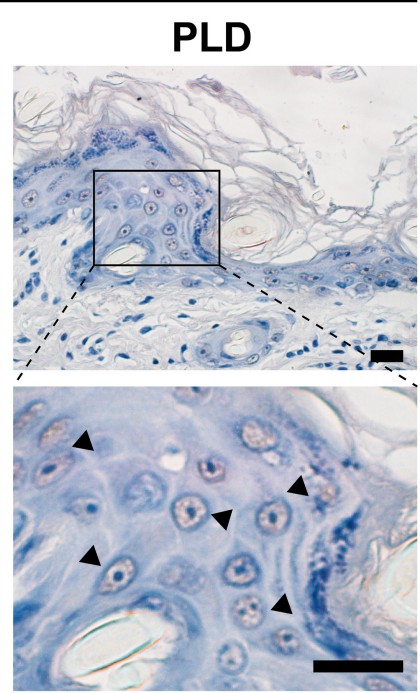

Figure 3 The IHC analysis of pro-inflammatory cytokines IL-6 and IL-I $\beta$ in the skin tissues of rats after the administration of PLD. Black arrows in the lower panel indicated the presence of positive cells. Scale bar: $20 \mu \mathrm{m}$.

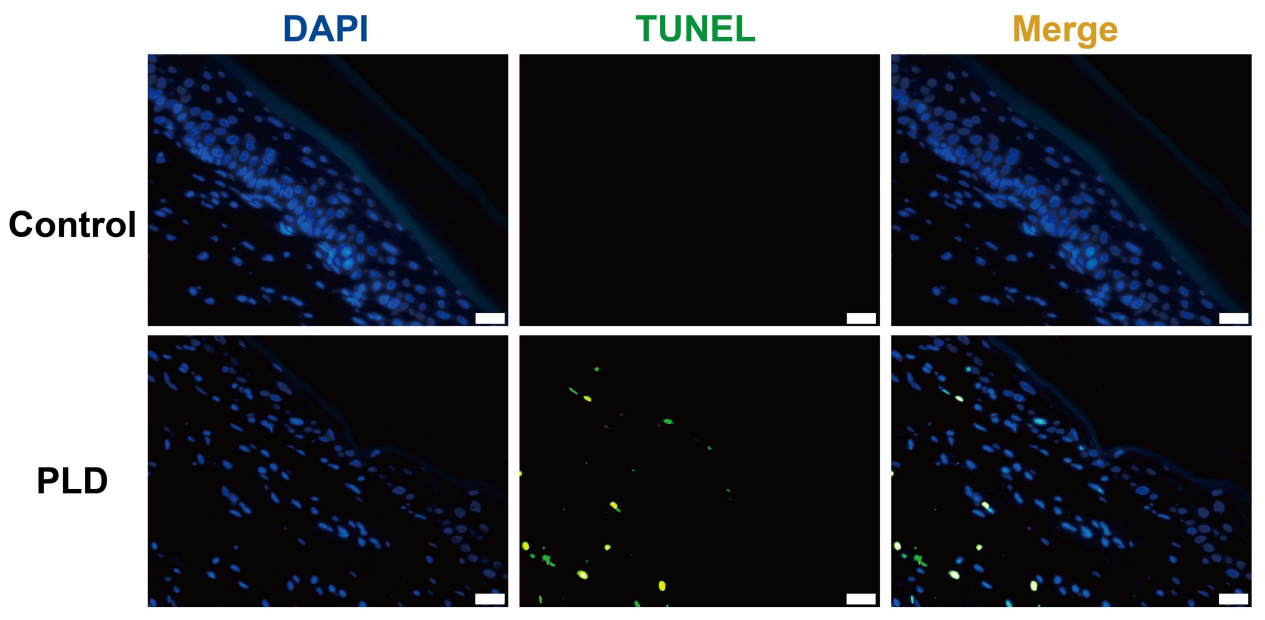

Figure 4 The apoptosis detection of skin tissues after the administration of PLD by TUNEL staining assay. DAPI staining (blue) represented the nucleus and TUNEL signal (green) indicated the occurrence of cell apoptosis. Scale bar: $20 \mu \mathrm{m}$.

free DOX, the PLD treatment exhibited less toxicity to cells, owing to the decreased cellular uptake of drugs after the introduction of PEG. Besides, the controlled release profile of PLD might attribute to the reduced anti-proliferation effect of nanomedicine. Since the viability values could be reduced to $<80.0 \%$ at a dose of $2.0 \mu \mathrm{M}$, this concentration was selected for the cell apoptosis and ROS level analysis in vitro. To further elucidate the inhibition of cell proliferation in $\mathrm{HaCaT}$ cells, the cell apoptosis was detected by TUNEL staining and flow cytometry (FACS) analysis based on the Annexin V-FITC and PI staining. As presented in Figure
$5 \mathrm{~A}$, no green fluorescence was observed in the control group, while the cells displayed obvious green signal after the treatment with DOX and PLD, indicating that both DOX and PLD could induce the cell apoptosis of keratinocytes. Particularly, more apoptotic cells were found in the DOX treatment group than PLD, which was consistent with the MTT analysis. This phenomenon also demonstrated that free small molecules such as DOX were much easier to penetrate the cell membrane than the nano-formulations, thereby triggering stronger cell apoptosis. Further, the apoptotic ratios of $\mathrm{HaCaT}$ cells were measured by FACS analysis after the staining 

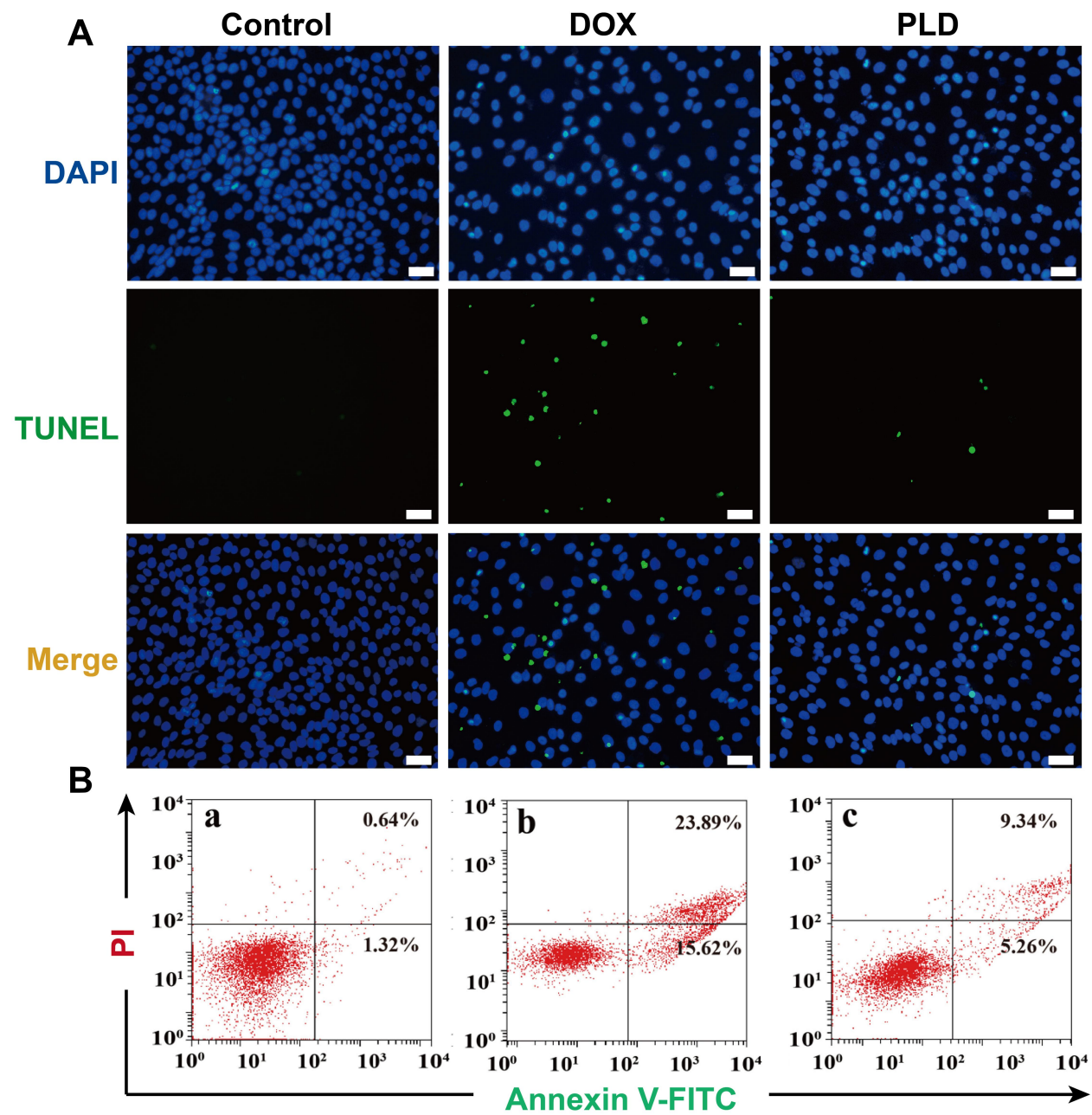

Figure 5 (A) The apoptosis detection in HaCaT cells by TUNEL staining assay. Blue (DAPI, nucleus), green (TUNEL). Scale bar: $20 \mu \mathrm{m}$. (B) The quantitative analysis of cell apoptosis after the treatment with DOX (b) and PLD (c) for 48 h by FACS, and no treatment group was used as the control (a).

with Annexin V-FITC and PI (Figure 5B). Apparently, compared to the control group, the percentage of apoptotic cells were significantly improved after the treatment with DOX or PLD for $48 \mathrm{~h}$, in which apoptotic ratios of $39.51 \%$ and $14.60 \%$ could be achieved in DOX and PLD treatment groups, respectively. These results elucidated that the injection of PLD could not only induce inflammation in the skin tissues but also cause obvious cell apoptotic effect, owing to the dermal accumulation of PLD and the subsequent DOX release in the keratinocytes.

\section{ROS Production in the Skin Tissues Induced by PLD}

To further investigate whether the inflammation and apoptosis induced by PLD was associated with the oxidative stress damage of DOX, the ROS production in the skin tissues was detected using FACS. As shown in Figure $6 \mathrm{~A}$ and $\mathrm{B}$, compared with the normal skin tissues, the damaged skin induced by the PLD administration exhibited a higher ROS level, indicating that the PLDinducing skin lesion was highly associated with the ROS generation. Additionally, we also measured the ROS production in $\mathrm{HaCaT}$ cells after the treatment with DOX and PLD (Figure 6C and D). The generation of ROS was clearly observed when the cells were incubated with DOX and PLD, and the DOX-treating cells produced higher ROS level than those receiving PLD. Thus, we concluded that PLD could achieve the controlled release of DOX and further lead to the ROS production in the keratinocytes to execute the oxidative stress damage. In the HFS model induced by the PLD administration, the extravasation of PLD could facilitate the dermal accumulation of drugs during the long-term circulation. Further, 
A

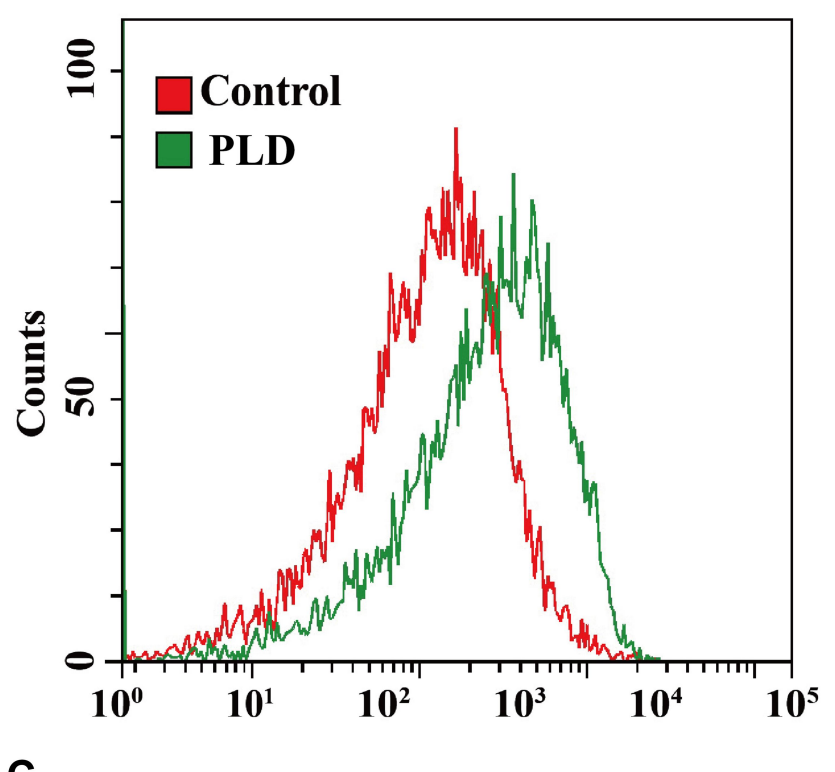

C

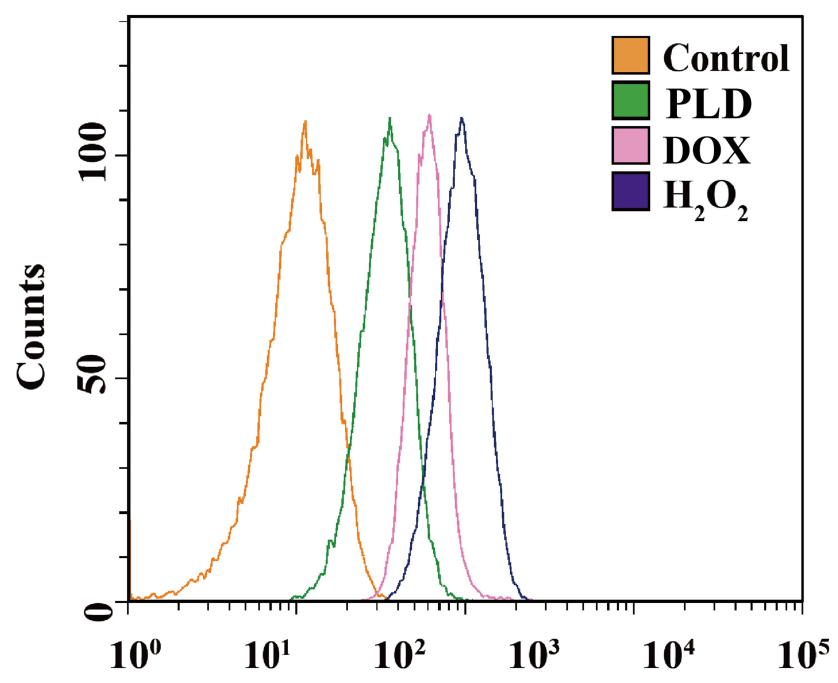

B
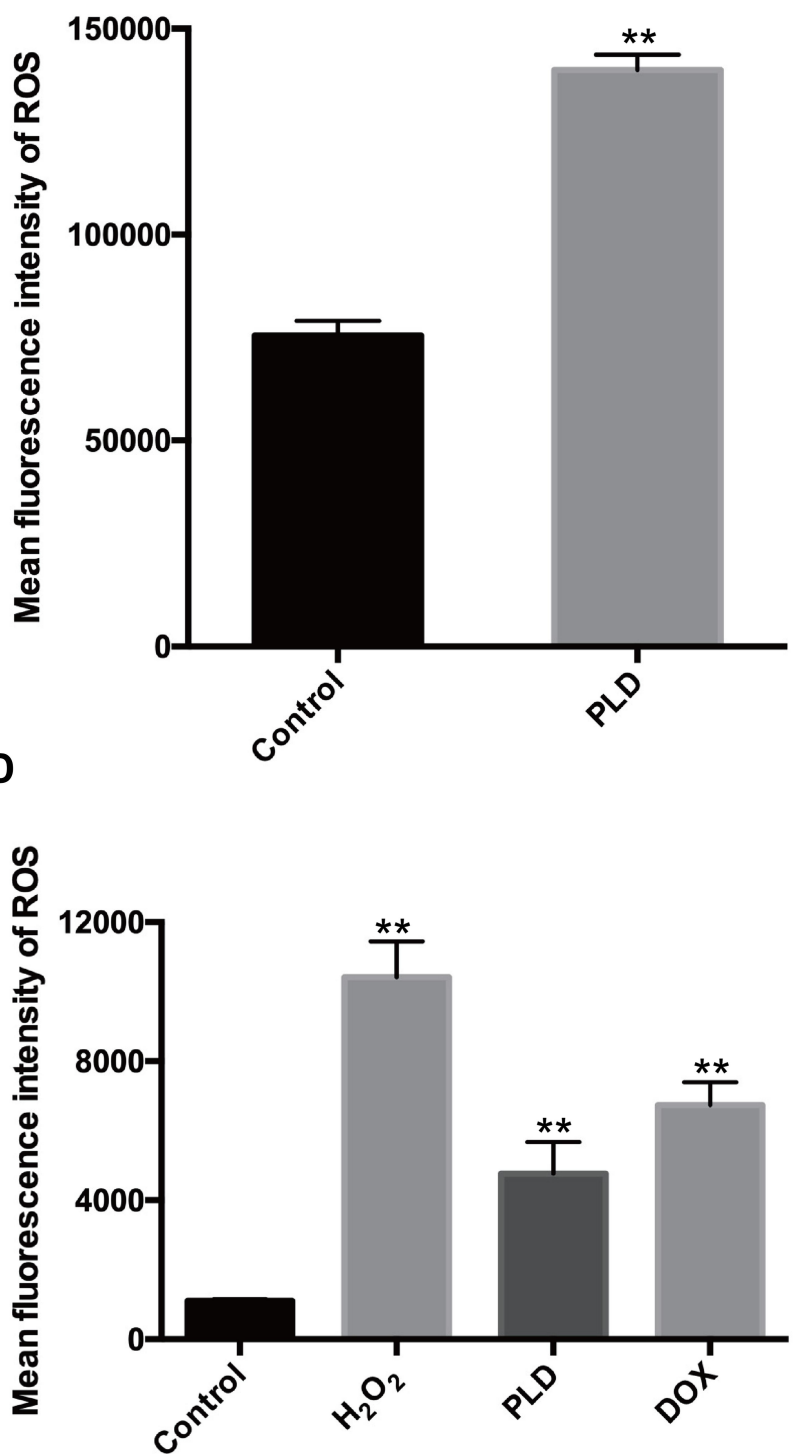

Figure 6 The ROS production in skin tissues and HaCaT cells detected by FACS ( $\mathbf{A}$ and $\mathbf{C})$ and the quantitative measurements based on the mean fluorescence intensity (B and D). Data were expressed as mean value \pm SD $(* * p<0.01)$.

the leaking PLD would release DOX in a sustained manner, which could interact with the copper ions abundant in the skin tissues, thus promoting the ROS generation in the keratinocytes. ${ }^{18}$ The ROS production triggered the oxidative stress and further induced the cell apoptosis of keratinocytes through many cellular pathways, such as the involvement of $\mathrm{p} 38 / \mathrm{p} 53$ and NF- $\mathrm{KB}$ pathway and the oxidative modification of ERK and Bcl-2 ubiquitination. $^{26-28}$ Meanwhile, the excess ROS level simultaneously mediated the production of proinflammatory cytokines such as IL-1 $\beta$ and IL-6, promoting an inflammatory induction in skin tissues. ${ }^{29}$ Moreover, the production of ROS also demonstrated the ability to destroy the collagen fibers which were the key components in the skin architecture. ${ }^{18}$

\section{Conclusion}

In conclusion, the intravenous administration of PLD has been demonstrated to induce severe skin lesions, which was a typical symptom of HFS. The phenomenon was probably caused by the accumulation of PLD in the skin tissues and the further penetration of capillary walls to reach the stratum corneum of skins, owing to its extravasation during the long-term circulation. The DOX released from PLD could interact with copper ions in the skin to generate ROS and then trigger the inflammation and 
apoptosis of cells, thereby yielding the damage of collagen fibers. Thus, the ROS level induced by the PLD administration was a key factor in the development of HFS, and the ROS generation could be an ideal target to alleviate the HFS symptom of PLD administration, such as the usage of antioxidative reagents including superoxide dismutase.

\section{Acknowledgments}

The authors gratefully acknowledge the support from National Natural Science Foundation of China (81673502 and 81872928), Education Department of Jilin Province (JJKH20190010KJ), and Province-University Cooperation Project of Jilin Province (SXGJQY2017-4).

\section{Disclosure}

The authors report no conflicts of interest in this work.

\section{References}

1. Barenholz Y. Doxil ${ }^{\circledR}$ - the first FDA-approved nano-drug: lessons learned. $J$ Control Release. 2012;160:117-134. doi:10.1016/j. jconrel.2012.03.020

2. Zhao Y, Alakhova DY, Kim JO, et al. A simple way to enhance Doxil $^{\circledR}$ therapy: drug release from liposomes at the tumor site by amphiphilic block copolymer. J Control Release. 2013;168:61-69. doi:10.1016/j.jconrel.2013.02.026

3. Green AE, Rose PG. Pegylated liposomal doxorubicin in ovarian cancer. Int J Nanomed. 2006;1:229-239.

4. Wu D, Si M, Xue H, et al. Nanomedicine applications in the treatment of breast cancer: current state of the art. Int $J$ Nanomed. 2017;12:5879-5892. doi:10.2147/IJN.S123437

5. Luo D, Carter KA, Molins EAG, et al. Pharmacokinetics and pharmacodynamics of liposomal chemophototherapy with short drug-light intervals. $J$ Control Release. 2019;297:39-47. doi:10.1016/j. jconrel.2019.01.030

6. Alimohammadi R, Alibeigi R, Nikpoor AR, et al. Encapsulated checkpoint blocker before chemotherapy: the optimal sequence of anti-CTLA-4 and Doxil combination therapy. Int $J$ Nanomed. 2020;15:5279-5288. doi:10.2147/IJN.S260760

7. Gabizon AA, Patil Y, La-Beck NM. New insights and evolving role of pegylated liposomal doxorubicin in cancer therapy. Drug Resist Updat. 2016;29:90-106. doi:10.1016/j.drup.2016.10.003

8. You S, Zuo L, Li W. Optimizing the time of doxil injection to increase the drug retention in transplanted murine mammary tumors. Int J Nanomed. 2010;5:221-229. doi:10.2147/IJN.S8488

9. Patel J, Ringley JT, Moore DC. Case series of docetaxel-induced dorsal hand-foot syndrome. Ther Adv Drug Saf. 2018;9:495-498. doi:10.1177/2042098618776911

10. Chen YH, Lee YT, Wen CC, et al. Modeling pegylated liposomal doxorubicin-induced hand-foot syndrome and intestinal mucositis in zebrafish. OncoTargets Ther. 2014;7:1169-1175. doi:10.2147/OTT. S63785

11. Swenson KK, Bell EM. Hand-foot syndrome related to liposomal doxorubicin. Oncol Nurs Forum. 2010;37:137-139. doi:10.1188/10. ONF.137-139

12. Alibolandi M, Abnous K, Mohammadi M, et al. Extensive preclinical investigation of polymersomal formulation of doxorubicin versus Doxil-mimic formulation. J Control Release. 2017;264:228-236. doi:10.1016/j.jconrel.2017.08.030
13. Nikolaou V, Syrigos K, Saif MW. Incidence and implications of chemotherapy related hand-foot syndrome. Expert Opin Drug Saf. 2016;15:1625-1633. doi:10.1080/14740338.2016.1238067

14. Lorusso D, Di Stefano A, Carone V, et al. Pegylated liposomal doxorubicin-related palmar-plantar erythrodysesthesia ('hand-foot' syndrome). Ann Oncol. 2007;18:1159-1164. doi:10.1093/annonc/ md1477

15. Miller KK, Gorcey L, McLellan BN. Chemotherapy-induced hand-foot syndrome and nail changes: a review of clinical presentation, etiology, pathogenesis, and management. $J$ Am Acad Dermatol. 2014;71:787-794. doi:10.1016/j.jaad.2014.03.019

16. He H, Jiang S, Xie Y, et al. Reassessment of long circulation via monitoring of integral polymeric nanoparticles justifies a more accurate understanding. Nanoscale Horiz. 2018;3:397-407. doi:10.1039/ C8NH00010G

17. Boers-Sonderen MJ, van Herpen CM, van der Graaf WT, et al. Correlation of toxicity and efficacy with pharmacokinetics (PK) of pegylated liposomal doxorubicin (PLD) $\left(\right.$ Caelyx $\left.^{\circledR}\right)$. Cancer Chemother Pharmacol. 2014;74:457-463. doi:10.1007/s00280-0142514-9

18. Yokomichi N, Nagasawa T, Coler-Reilly A, et al. Pathogenesis of hand-foot syndrome induced by PEG-modified liposomal doxorubicin. Hum Cell. 2013;26:8-18. doi:10.1007/s13577-0120057-0

19. Barygina V, Becatti M, Lotti T, et al. ROS-challenged keratinocytes as a new model for oxidative stress-mediated skin diseases. $J$ Cell Biochem. 2019;120:28-36. doi:10.1002/jcb.27485

20. Liang $X$, Tang $X$, Yang $J$, et al. A genipin-crosslinked protein-polymer hybrid system for the intracellular delivery of ribonuclease A. Int J Nanomed. 2019;14:7389-7398. doi:10.2147/IJN. S210486

21. Jia J, Zhang S, Wen K, et al. Nano-scaled zeolitic imidazole framework-8 as an efficient carrier for the intracellular delivery of RNase A in cancer treatment. Int J Nanomed. 2019;14:9971-9981. doi:10.2147/IJN.S210107

22. Schmook T, Jacobi U, Lademann J, et al. Detection of doxorubicin in the horny layer in a patient suffering from palmar-plantar erythrodysaesthesia. Dermatology. 2005;210:237-238. doi:10.1159/ 000083794

23. Jacobi U, Waibler E, Schulze P, et al. Release of doxorubicin in sweat: first step to induce the palmar-plantar erythrodysesthesia syndrome? Ann Oncol. 2005;16:1210-1211. doi:10.1093/annonc/ mdi204

24. Matos TR, de Rie MA. Discovery of skin lymphocytes was a game changer in experimental dermatology. Exp Dermatol. 2017;26:683-684. doi:10.1111/exd.13320

25. Cui SJ, Fu Y, Liu Y, et al. Chronic inflammation deteriorates structure and function of collagen fibril in rat temporomandibular joint disc. Int J Oral Sci. 2019;11:2. doi:10.1038/s41368-018-0036-8

26. Yang J, Qiao L, Zeng Z, et al. The role of the ATM/Chk/P53 pathway in mediating DNA damage in hand-foot syndrome induced by PLD. Toxicol Lett. 2017;265:131-139. doi:10.1016/j.toxlet.2016.11.024

27. Pelin M, Fusco L, Martin C, et al. Graphene and graphene oxide induce ROS production in human $\mathrm{HaCaT}$ skin keratinocytes: the role of xanthine oxidase and $\mathrm{NADH}$ dehydrogenase. Nanoscale. 2018;10:11820-11830. doi:10.1039/C8NR02933D

28. Luanpitpong S, Chanvorachote P, Nimmannit U, et al. Mitochondrial superoxide mediates doxorubicin-induced keratinocyte apoptosis through oxidative modification of ERK and Bcl-2 ubiquitination. Biochem Pharm. 2012;83:1643-1654. doi:10.1016/j.bcp.2012.03.010

29. Ryu YS, Kang KA, Piao MJ, et al. Particulate matter induces inflammatory cytokine production via activation of NF- $\mathrm{kB}$ by TLR5-NOX4 -ROS signaling in human skin keratinocyte and mouse skin. Redox Biol. 2019;21:101080. doi:10.1016/j.redox.2018.101080 


\section{Publish your work in this journal}

The International Journal of Nanomedicine is an international, peerreviewed journal focusing on the application of nanotechnology in diagnostics, therapeutics, and drug delivery systems throughout the biomedical field. This journal is indexed on PubMed Central, MedLine, CAS, SciSearch ${ }^{\mathbb{R}}$, Current Contents ${ }^{\mathbb{R}} /$ Clinical Medicine, $^{2}$
Journal Citation Reports/Science Edition, EMBase, Scopus and the Elsevier Bibliographic databases. The manuscript management system is completely online and includes a very quick and fair peer-review system, which is all easy to use. Visit http://www.dovepress.com/ testimonials.php to read real quotes from published authors. 